\title{
Erratum to: The comparative efficacies of intra-articular and IV tranexamic acid for reducing blood loss during total knee arthroplasty
}

\author{
Jai-Gon Seo • Young-Wan Moon • Sang-Hoon Park • \\ Sang-Min Kim • Kyung-Rae Ko
}

Published online: 6 February 2013

(c) Springer-Verlag Berlin Heidelberg 2013

Erratum to: Knee Surg Sports Traumatol Arthrosc

DOI 10.1007/s00167-012-2079-2

Unfortunately, data in Table 3 were incorrectly published in the original article. The corrected data are given below.

Table 3 Postoperative complications

\begin{tabular}{llll}
\hline & $\begin{array}{l}\text { Intra-articular } \\
\text { injection group } \\
(n=50)\end{array}$ & $\begin{array}{l}\text { IV injection } \\
\text { group } \\
(n=50)\end{array}$ & $\begin{array}{l}\text { Placebo } \\
\text { group } \\
(n=50)\end{array}$ \\
\hline $\begin{array}{l}\text { Number of DVT } \\
\text { Number of atrial } \\
\text { fibrillation }\end{array}$ & 0 & 3 & 2 \\
$\begin{array}{l}\text { Number of } \\
\text { pulmonary }\end{array}$ & 0 & 1 & 1 \\
embolism & & 0 & 0 \\
\hline
\end{tabular}

DVT deep vein thrombosis

The online version of the original article can be found under doi:10.1007/s00167-012-2079-2.

J.-G. Seo · Y.-W. Moon · S.-M. Kim · K.-R. Ko

Department of Orthopaedic Surgery, Samsung Medical Center, Sungkyunkwan University School of Medicine,

Seoul, South Korea

S.-H. Park $(\square)$

Department of Orthopaedic Surgery, National Health Insurance

Corporation Ilsan Hospital, Ilsan-ro 100, Ilsandong-gu,

Goyang, Kyunggi-do 410-719, South Korea

e-mail: orthomania@gmail.com 